Rev. Int. Contam. Ambie. 33 (Especial Biotecnología e ingeniería ambiental) 7-21, 2017

DOI: 10.20937/RICA.2017.33.esp01.01

\title{
CONTAMINACIÓN AMBIENTAL POR METALES PESADOS EN MÉXICO: PROBLEMÁTICA Y ESTRATEGIAS DE FITORREMEDIACIÓN
}

\author{
Sergio Abraham COVARRUBIAS y Juan José PEÑA CABRIALES*
}

Departamento de Biotecnología y Bioquímica, Centro de Investigación y de Estudios Avanzados, Instituto Politécnico Nacional, Unidad Irapuato. Libramiento norte, Carretera Irapuato León, kilómetro 9.6, Irapuato, Guanajuato, México, C.P. 36821

*Autor para correspondencia: jpena@ira.cinvestav.mx

(Recibido abril 2015; aceptado octubre 2016)

Palabras clave: remediación, microorganismos, suelos

\section{RESUMEN}

La contaminación por metales pesados en México es un problema que va en aumento debido a la actividad antrópica, específicamente la minería. Los metales contaminantes más importantes en México, dada su toxicidad y abundancia son: mercurio, arsénico, plomo y cromo. Entre los sitios más afectados por las altas concentraciones de metales pesados en suelos se encuentran los estados de Zacatecas, Querétaro, Hidalgo y San Luis Potosí. Una alternativa para contribuir a la solución de este problema, es el uso de especies vegetales para la remoción de metales pesados del suelo o "fitorremediación". Al respecto, en México se han caracterizado especies con capacidad de acumulación de metales, como Scirpus americanus, Typha latifolia, Jatropha dioica, Eichhornia crassipes y Amaranthus hybridus. Una estrategia para mejorar el proceso de fitoextracción de metales es a través de la inoculación de microorganismos del suelo. Bacterias de los géneros Rhizobium, Agrobacterium, Arthrobacter, Microbacterium, Curtobacterium, Rhodococcus, Xanthomonas y Pseudomonas, han mostrado resultados promisorios al ser empleados en estos tratamientos, así como el uso de hongos micorrízicos arbusculares específicamente del género Glomus. Sin embargo, son necesarios más estudios para optimizar los sistemas de tratamiento basados en la selección de las mejores plantas y bacterias endémicas de los sitios contaminados.

Key words: remediation, microorganisms, soils

\begin{abstract}
Heavy metal pollution is a growing problem due to anthropic activities such as mining. The most hazardous metals in Mexico are mercury, arsenic, lead, and chromium. States like Zacatecas, Querétaro, Hidalgo, and San Luis Potosi have been affected by the pollution of soils with heavy metals. In this sense, phytoremediation has emerged as an alternative to solve this problem. In Mexico, plant species with metal accumulative capacity have been identified. These include Scirpus americanus, Typha latifolia, Jatropha dioica, Eichhornia crassipes and Amaranthus hybridus. Metal phytoextraction could be improved by coupling bioaugmentation of soil microorganism. Bacteria from the genus Rhizobium, Agrobacterium, Arthrobacter, Microbacterium, Curtobacterium, Rhodococcus, Xanthomonas and Pseudomonas, have shown promising results enhancing
\end{abstract}


heavy metal phytoextraction, as well as the arbuscular fungi from the Glomus genus. However, more studies on endemic plants and microbial from contaminated sites are needed to optimize current treatment strategies.

\section{INTRODUCCIÓN}

La contaminación por metales pesados es un problema que ha ido en aumento debido principalmente a actividades antrópicas. Entre las principales fuentes de contaminación se encuentran la minería, la metalúrgica, la agricultura, los vehículos automotores y el aporte natural en ciertos acuíferos.

En México, existen reportes de la presencia de metales pesados en ríos, lagos, cultivos, suelos y aire de zonas urbanas, así como en ambientes costeros y marinos, donde se ha detectado la acumulación de metales tóxicos en tejidos de peces y moluscos de consumo humano (Villanueva y Botello 1992, García-Hernández et al. 2007, González-Dávila et al. 2012).

La minería es una de las principales causas de la contaminación ambiental por metales pesados, debido principalmente al manejo inadecuado de sus residuos denominados "jales mineros", lo que ocasiona problemas de contaminación en estados como Zacatecas, San Luis Potosí, Guerrero y Sonora. (Yañez et al. 2003, Meza-Figueroa et al. 2009, Mireles et al. 2012, Cortés-Jiménez et al. 2013).

Reportes indican que en México podrían existir millones de toneladas de jales dispersos en el territorio nacional, de los que todavía se desconocen sus condiciones y su potencial de afectación al ambiente (Ramos-Arroyo y Siebe-Grabach 2006).

El objetivo de esta revisión es analizar la problemática de contaminación por metales pesados en México, enfocándose en aquellos más abundantes y discutir el uso de la fitorremediación asistida por microorganismos como una alternativa biológica para ayudar a la solución de este problema.

\section{METALES PESADOS Y METALOIDES}

Los metales pesados son un conjunto de elementos que presentan propiedades metálicas, en el que se incluyen metales de transición, algunos semimetales, lantánidos y actínidos. El criterio más general para su clasificación se basa en la densidad específica, de acuerdo con la cual se incluyen en este grupo elementos con una densidad mayor a $5 \mathrm{~g} / \mathrm{cm}^{3}$. Sin embargo, algunos autores consideran esta definición como inapropiada, pues la densidad específica no es una referencia en cuanto a la reactividad o toxicidad de un metal (Duffus 2002, Volke-Sepulveda et al. 2005).

Actualmente, el término "metal pesado" es utilizado para referirse de una manera amplia a aquellos metales o metaloides con potencial de causar problemas de toxicidad (Alloway 2013). Los principales mecanismos de toxicidad a nivel molecular son:

1. Bloqueo de grupos funcionales esenciales en biomoléculas, debido a la alta afinidad de los cationes metálicos por los grupos sulfhidrilos de las proteínas, específicamente a los residuos de cisteína, lo que ocasiona su desnaturalización (Schützendübel y Polle 2002, Peralta-Videa et al. 2009).

2. El desplazamiento de centros catiónicos en enzimas importantes, como es el caso de la ribulosa 1-5 bisfosfato carboxilasa-oxigenasa (rubisco), la cual tiene un centro catiónico de $\mathrm{Mg}^{2+}$ que puede ser desplazado en presencia de cationes divalentes como $\mathrm{Co}^{2+}, \mathrm{Ni}^{2+}$ y $\mathrm{Zn}^{2+}$. Dicho desplazamiento provoca que la mencionada enzima pierda su función (Schützendübel y Polle 2002, Smeets et al. 2005).

3. Por la formación de especies reactivas de oxígeno (ERO) debido a la autooxidación de metales como $\mathrm{Fe}^{2+}$ o $\mathrm{Cu}^{+}$, lo que resulta en la formación de $\mathrm{H}_{2} \mathrm{O}_{2}$ y del radical $\cdot \mathrm{OH}$ vía reacción de Fenton (Schützendübel y Polle 2002). El radical $\cdot \mathrm{OH}$ es uno de los más reactivos que se conocen, por su capacidad de iniciar reacciones en cadena de radicales libres que ocasionan modificaciones y daño irreversible a compuestos celulares como carbohidratos, ácido desoxirribonucleico (ADN), proteínas y particularmente lípidos (Mithöfer et al. 2004).

\section{LA MINERÍA EN MÉXICO Y LOS PRINCIPALES CONTAMINANTES METÁLICOS}

En México, la minería constituye una de las actividades con mayor tradición, practicada desde la época prehispánica y reconocida en la historia como un factor de avance y desarrollo económico para el país. Sin embargo, esta actividad tiene un impacto 
considerable sobre el ambiente ya que genera grandes cantidades de desechos que pueden convertirse en fuentes de metales pesados (Romero et al. 2007).

El ejemplo más común es la contaminación de suelos que ocurre durante el beneficio del oro y la plata, realizado comúnmente por amalgamación con mercurio y cianuración. En ninguno de los dos casos es posible la recuperación total de los compuestos o elementos adicionados, por lo que es común encontrarlos en los residuos del proceso (jales mineros) en forma soluble (Volke-Sepulveda et al. 2005). Debido a lo anterior los jales contienen una gran cantidad de metales residuales que derivan de un proceso de extracción que no es $100 \%$ eficiente.

Los principales metales contaminantes en México, considerando su toxicidad y abundancia son: mercurio $(\mathrm{Hg})$, arsénico $(\mathrm{As})$, plomo $(\mathrm{Pb})$ y en algunos lugares el cromo $(\mathrm{Cr})$ (Fernández-Bremauntz et al. 2004, Volke-Sepulveda et al. 2005, Romero y Gutiérrez-Ruiz 2010). En el cuadro I se muestran los límites máximos permitidos de estos metales para suelos y aguas en México, establecidos en las normas NOM-147-SEMARNAT/SSA1-2004 (SEMARNAT 2007) y NOM-001-SEMARNAT-1996 (SEMARNAT 2003) respectivamente. A continuación, se describen las generalidades de estos contaminantes.

\section{Mercurio}

El mercurio puede llegar al ambiente a través de procesos naturales como la actividad volcánica, depósitos minerales, incendios forestales, emisiones oceánicas y desgasificación de la corteza terrestre (Ruelas-Inzunza et al. 2011). Sin embargo, el mayor aporte proviene de las actividades antrópicas, principalmente la minería y la combustión industrial. A diferencia de otros metales el mercurio se encuentra presente en la atmósfera en estado gaseoso (de la Rosa et al. 2004, Li y Tse 2015), lo cual facilita su transporte a través de la biósfera.

Los efectos adversos del mercurio sobre la salud humana varían de acuerdo con su forma química, concentración y tiempo de exposición. La forma más tóxica es el metil mercurio $\left[\mathrm{CH}_{3} \mathrm{Hg}\right]^{+}$, ya que se absorbe en un $90 \%$ comparado con el cloruro de mercurio $\left(\mathrm{HgCl}_{2}\right)$ del cual solamente se absorbe un $2 \%$ (UNEP 2013). Algunos de los efectos en la salud consisten en daños a diferentes órganos, principalmente el cerebro y los riñones, además de causar trastornos al sistema nervioso (Li et al. 2011).

Durante la época colonial, se trajeron de España grandes cantidades de mercurio para utilizarlo en el proceso de amalgamación de los metales en minas de extracción de plata localizadas en Zacatecas, Guanajuato y San Luis Potosí. Fue durante el periodo colonial y hasta mediados del siglo XX que se depositaron grandes cantidades de mercurio al ambiente como consecuencia de la ausencia de normativas y procesos apropiados para el manejo de residuos (Castro-Díaz 2011).

La contaminación por mercurio no afecta solamente al suelo, este problema se presenta también en ambientes acuáticos debido a las descargas antrópicas. En este caso el mercurio puede ser bioacumulado a través de la cadena alimenticia en forma de metilmercurio (García-Hernández et al. 2007), el cual resulta muy peligroso para el humano, en especial para los niños en gestación.

\section{Arsénico}

En la naturaleza, el arsénico se encuentra principalmente en formas inorgánicas como el arsenato [As (V)] y la arsenita [As (III)] (Prohaska y Stingeder 2005). Bajo condiciones aeróbicas el arsenato tiene una alta afinidad por oxihidróxidos de hierro $[\mathrm{FeO}(\mathrm{OH})]$ y óxidos de manganeso $\left(\mathrm{MnO}_{2}\right)$, lo que lo vuelve una forma relativamente inmóvil en suelos, mientras que la arsenita debido a su baja afinidad por estas formas tiene una mayor movilidad (Meharg y Zhao 2012).

El arsénico se puede presentar en variantes orgánicas, producidas por acción del metabolismo de microorganismos, plantas y mamíferos. Formas como

CUADRO I. LÍMITES MÁXIMOS PERMISIBLES DE METALES PESADOS Y ARSÉNICO EN SUELOS NOM147-SEMARNAT-SSA1-2004 (SEMARNAT 2007) Y EN AGUA NOM-001-SEMARNAT-1996 (SEMARNAT 2003)

\begin{tabular}{|c|c|c|c|c|}
\hline \multirow[t]{2}{*}{ Metal } & Suelo uso agrícola/re & \multirow[t]{2}{*}{ Suelo uso industrial } & Agua uso agrícola & Agua uso público \\
\hline & & & \multicolumn{2}{|c|}{$(\mathrm{mg} / \mathrm{L})$} \\
\hline As & 22 & 260 & 0.20 & 0.100 \\
\hline $\mathrm{Hg}$ & 23 & 310 & 0.01 & 0.005 \\
\hline $\mathrm{Pb}$ & 400 & 800 & 0.50 & 0.200 \\
\hline $\mathrm{Cr}(\mathrm{VI})$ & 280 & 510 & 1.00 & 0.500 \\
\hline
\end{tabular}


el ácido monometilarsénico, dimetilarsénico, arsenobetaina y arsenoazúcares resultan menos tóxicas que las inorgánicas (Singh y Ma 2006).

Algunos de los síntomas que se manifiestan debido a la ingestión crónica de arsénico son: cambios en la coloración de la piel, arterosclerosis, fallas renales, efectos neurológicos, enfermedades cardiovasculares, esterilidad y cáncer en piel, pulmones, hígado y riñones (Singh y Ma 2006).

\section{Plomo}

El plomo se encuentra presente en la corteza terrestre de forma natural y en pequeñas cantidades $(0.002 \%)$. Se obtiene generalmente a partir de la galena $(\mathrm{PbS})$, la anglesita $\left(\mathrm{PbSO}_{4}\right)$ y la curosita $\left(\mathrm{PbCO}_{3}\right)$ (Volke-Sepulveda et al. 2005).

El plomo al ser un catión divalente, se une de manera estrecha a los grupos sulfhidrilos de las proteínas ocasionando su desnaturalización. La exposición a dosis elevadas de este elemento puede ocasionar una serie de efectos adversos a la salud, que incluyen daño cerebral severo e incluso la muerte. En mujeres embarazadas puede provocar aborto, mientras que en hombres altera la espermatogénesis (ATSDR 2007).

Las principales fuentes de emisión de plomo al ambiente son la fundición y el procesamiento de metales, el reciclamiento de baterías ácidas de plomo, la minería a través de la disposición de jales mineros y la contaminación de la atmósfera debido al uso de gasolinas con plomo (Volke-Sepulveda et al. 2005). Aunado a lo anterior, es común que en los sitios contaminados por plomo se encuentren otros metales contaminantes como As, $\mathrm{Hg}$ y $\mathrm{Zn}$.

\section{Cromo}

El cromo puede existir en diferentes formas, dependiendo de su estado de oxidación, puede encontrarse en estado líquido, sólido o gaseoso. Las formas químicas más comunes son el $\mathrm{Cr}(0), \mathrm{Cr}$ (III) y $\mathrm{Cr}$ (VI), siendo este último su forma más tóxica (ATSDR 2012). En México la industria de la curtiduría es una de las que más aporta a la contaminación por este elemento, debido al uso de sales de cromo para el procesado de las pieles.

La ingestión de agua con altas cantidades de $\mathrm{Cr}$ (VI) puede ocasionar problemas intestinales, enfermedades gástricas y hepáticas. El Cr (VI) es también considerado un elemento genotóxico y citotóxico para bacterias y organismos eucariontes (RoblesCamacho y Armienta 2000), mientras que el Cr (III) debido a que se precipita a $\mathrm{pH}$ neutros, es mucho menos tóxico que el Cr (IV).

\section{PRINCIPALES SITIOS CONTAMINADOS CON METALES PESADOS EN MÉXICO}

Uno de los sitos afectados por la contaminación de metales pesados es la laguna "La Zacatecana", en el municipio de Guadalupe, Zacatecas. En esta zona desde la época virreinal hasta 1900 fueron depositados a través de los ríos que desembocaban en el área, los desechos del proceso de amalgamación de la plata, los cuales contenían altas concentraciones de plomo, arsénico y mercurio (Santos-Santos et al. 2006). Estos desechos se acumularon en la laguna la cual se deseca completamente durante una época del año y es aprovechada para la agricultura, representando un posible vector de contaminación.

La laguna cuenta con una extensión aproximada de 120 ha y ha sido objeto de estudios para determinar el grado de afectación por esta problemática (Iskander et al. 1994). Cálculos recientes estiman que a la fecha existen en el lugar entre 10 y 20 millones de toneladas de residuos mineros acumulados (Zetina-Rodríguez 2012). Actualmente en esta zona se cultivan importantes productos agrícolas de consumo humano como frijol y maíz, sobre los cuales existen reportes que indican la acumulación de arsénico y plomo (González-Dávila et al. 2012). Asimismo, se ha reportado la presencia de niveles elevados de plomo y cadmio en sangre, así como arsénico en orina de niños del lugar (Trejo-Acevedo et al. 2009).

En otro estudio en las ciudades de Zacatecas y Guadalupe, se determinó la concentración de metales pesados en el suelo urbano, encontrando concentraciones de arsénico que rebasan hasta cinco veces lo establecido por las normas mexicanas para este tipo de suelo (Mireles et al. 2012).

Otro de los lugares afectados por la contaminación de metales pesados es el municipio de Zimapán en el estado de Hidalgo, considerado un distrito minero desde 1576 (Ongley et al. 2007) y donde hasta la década de los 40 se encontraban en activo cerca de 40 hornos de fundición (Armienta et al. 1997b). Las altas concentraciones de arsénico presentes en el acuífero de Zimapán, exceden hasta 10 veces los estándares establecidos por la Organización Mundial de la Salud (Ongley et al. 2007). Esto se debe principalmente al aporte natural de arsénico ocasionado por la oxidación de minerales que contienen ese elemento, como la arsenopirita (Armienta et al. 2000), además de la contribución por la actividad minera debido a la existencia de grandes depósitos de jales mineros sin tratar (Espinosa et al. 2009). 
Esta problemática ha repercutido en la salud de la población local como lo señalan Armienta et al. (1997a), quienes determinaron que la concentración de arsénico en el cabello de los pobladores de Zimapán se encuentra por arriba de los estándares internacionales. Aunado a lo anterior, el $80 \%$ de los habitantes presentan cierto grado de afectación en la piel, correlacionado directamente con la concentración de As en el cabello.

Respecto a los ambientes acuáticos, se ha reportado la acumulación de mercurio en peces de consumo humano, en algunos casos se han encontrado concentraciones mayores a los límites establecidos en las normas nacionales $(1 \mathrm{mg} / \mathrm{kg})$ como es el caso específico del tiburón (García-Hernández et al. 2007). Otro ejemplo, es lo reportado por Velasco-González et al. (2001) quienes, al analizar la concentración de mercurio en seis diferentes marcas de atún enlatado producido en México, encontraron que el $36 \%$ de las muestras rebasaron la concentración de $\mathrm{Hg}$ establecida por las normas mexicanas.

Otro estado de la República Mexicana afectado por la contaminación de metales pesados es San Luis Potosí. En el poblado minero de Villa de la Paz se detectaron concentraciones elevadas de plomo y arsénico en sangre y orina de los niños del lugar (Yañez et al. 2003). Lo anterior está relacionado con las altas concentraciones de estos metales en el suelo. En un estudio reciente realizado en la ciudad de San Luis Potosí, se determinó la cantidad de As, $\mathrm{Cd}, \mathrm{Pb}$ y $\mathrm{Hg}$ en suelo. Se encontró que en todos los sitios estudiados la concentración de As rebasó los límites permitidos por las normas (Perez-Vazquez et al. 2015).

En el cuadro II se mencionan otros casos reportados en la literatura donde existe una problemática por contaminación con metales pesados.

\section{BIORREMEDIACIÓN EN MÉXICO}

A pesar de la legislación existente sobre la disposición y manejo de residuos, es evidente que el problema persiste. Lo anterior, aunado al remanente de desechos históricos de la minería, agrava la situación de la contaminación por metales pesados en México.

Diversas instituciones públicas han desarrollado investigaciones para establecer la magnitud de este problema y han propuesto estrategias para contribuir a la solución del mismo, enfocándose en el uso de alternativas biológicas que resultan en una menor alteración del ambiente, específicamente a través del uso de plantas para la remoción de metales pesados o "fitorremediación".

\section{FITORREMEDIACIÓN}

Las plantas al ser expuestas a metales pesados pueden presentar diferentes respuestas fisiológicas, las cuales varían dependiendo de la especie de la planta, el metal específico al que es expuesta y la concentración en el suelo del mismo. Con base en dichas respuestas las plantas pueden clasificarse en tres tipos: excluyentes, indicadoras y acumuladoras de metales pesados (Baker 1981).

a) Excluyentes: son aquellas plantas donde la acumulación de metales en la parte aérea es mucho menor respecto a la concentración de metales en el suelo.

b) Indicadoras: aquellas plantas donde la acumulación de metales en el tejido aéreo guarda una relación lineal respecto a la concentración del suelo.

c) Acumuladoras: plantas donde la acumulación de metales en su parte aérea es mucho mayor que la concentración de metales en el suelo.

Con base en la clasificación anterior y dependiendo del proceso de remediación que se desee utilizar, la fitorremediación de suelos contaminados por metales pesados presenta dos vertientes: la fitoestabilización y la fitoextracción.

En la fitoestabilización se buscan especies del tipo excluyente, que acumulen gran cantidad de metales en la raíz, evitando que estos puedan transferirse a la parte aérea y así estar disponibles para otros seres vivos. En cambio, en la fitoextracción se buscan especies del tipo acumuladoras, donde el contaminante sea transportado desde la raíz hasta los brotes aéreos, para que después pueda ser cosechado y confinado. La fitoextracción es hasta la fecha uno de los campos con mayores avances científicos y tecnológicos (Volke-Sepulveda et al. 2005).

Para determinar si una especie vegetal podría ser utilizada en un tratamiento de fitorremediación, se tienen que tomar en cuenta los siguientes parámetros: el factor de bioconcentración (FBC) y el factor de traslocación (FT) (Olguín y Sánchez-Galván 2012, Ali et al. 2013).

El FBC determina la eficiencia de la planta para acumular el metal proveniente del suelo en su tejido y se calcula de la siguiente manera:

$\mathrm{FBC}=$ concentración de metal en tejido/ concentración de metal en el suelo

Mientras que el FT indica la eficiencia para transportar el metal de la raíz a su parte aérea, se calcula de la siguiente manera: 


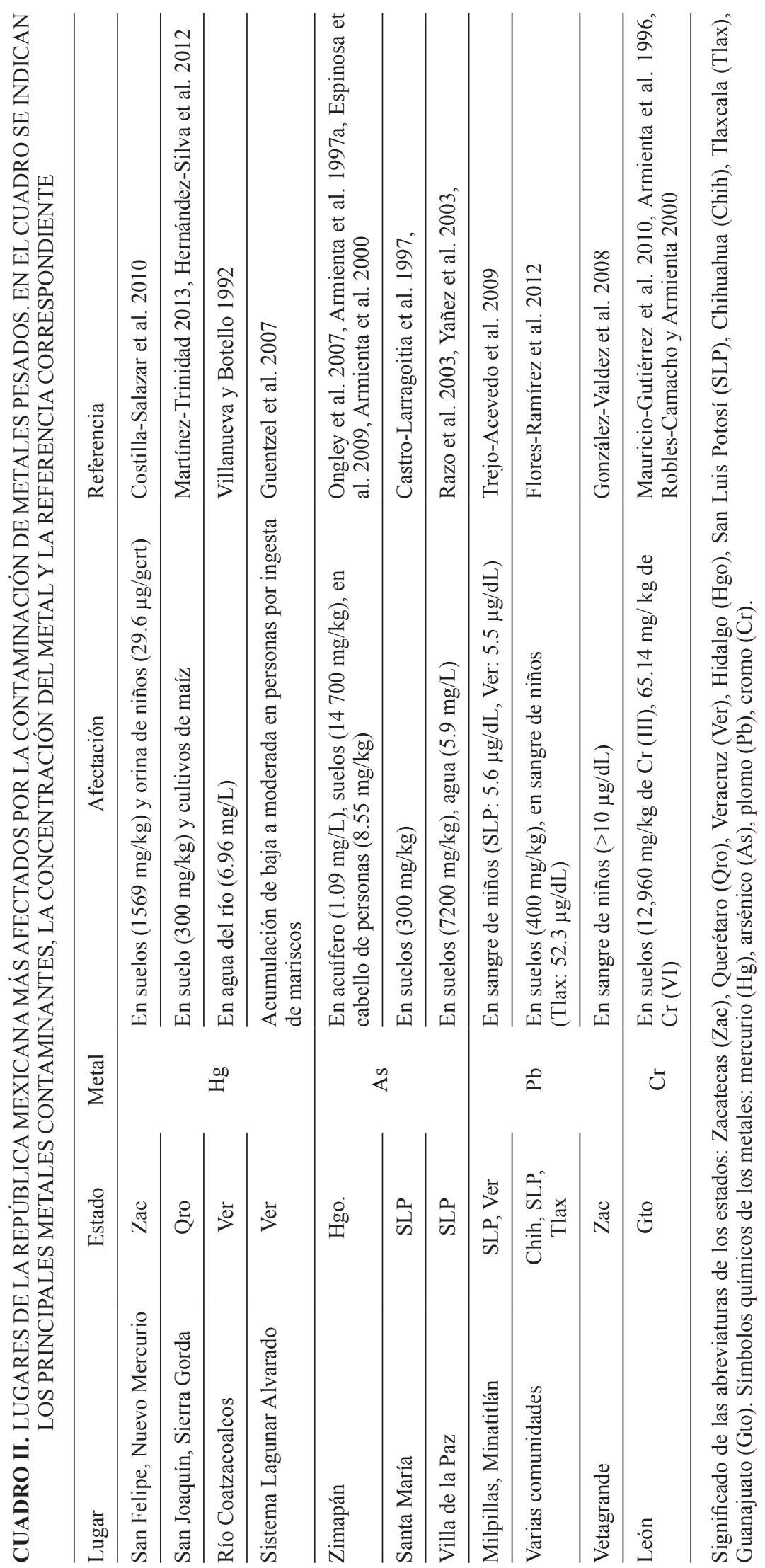


$\mathrm{FT}=$ concentración de metal en la parte aérea/concentración de metal en la raíz

Una planta puede considerarse acumuladora si su FT es igual o mayor a 1 , mientras aquellas que tienen la capacidad de acumular desde 5 hasta 500 veces más que el promedio se les denomina "hiperacumuladoras” (Pilon-Smits 2005, Rascio y Navari-Izzo 2011).

A nivel mundial se conocen cerca de 400 especies de plantas hiperacumuladoras de metales, destacando la familia Brassicaceae, por contar con varias especies que pueden acumular más de un tipo de metal (Prasad y Freitas 2003, Gratao et al. 2005).

\section{ESPECIES VEGETALES ACUMULADORAS DE METALES EN MÉXICO}

El primer paso para establecer un sistema de fitoextracción exitoso es encontrar especies vegetales que tengan la capacidad para acumular metales en su raíz y transportarlos a su tejido aéreo. En los tratamientos de fitorremediación las posibilidades de éxito aumentan al utilizar especies endémicas de cada sitio contaminado, debido a que se encuentran mejor adaptadas a las condiciones climáticas del lugar y a la mezcla específica de contaminantes de cada sitio en particular.

En México se han realizado inventarios florísticos de plantas que crecen en sitos contaminados por metales pesados (Cuadro III). En el análisis destacan algunas especies como Prosopis leavigata y Acacia farnesiana, las cuales son especies obligadas en lugares contaminados de zonas áridas y semiáridas, capaces de acumular en tallo hasta 87 y $225 \mathrm{mg} / \mathrm{kg}$ de arsénico, respectivamente (Armienta et al. 2008).

Por otro lado, Typha latifolia y Scirpus americanus son especies comúnmente utilizadas para la

CUADRO III. ESPECIES DE PLANTAS ACUMULADORAS DE METALES PESADOS, CRECIENDO EN DIFERENTES LUGARES DE MÉXICO

\begin{tabular}{|c|c|c|c|c|}
\hline Especie & Metal & Lugar & Estado & Referencia \\
\hline Prosopis laevigata & As & Zimapán & Hgo & \multirow[t]{2}{*}{ Armienta et al. 2008} \\
\hline Acacia farnesiana & As & Zimapán & Hgo & \\
\hline Scirpus americanus & $\mathrm{Pb}, \mathrm{Cd}, \mathrm{Cr}, \mathrm{Mn}$ & Tanque Tenorio & SLP & \multirow[t]{2}{*}{ Carranza-Álvarez et al. 2008} \\
\hline Typha latifolia & $\mathrm{Pb}, \mathrm{Cd}, \mathrm{Cr}, \mathrm{Mn}$ & Tanque Tenorio & SLP & \\
\hline Polygonum aviculare & $\mathrm{Zn}$ & El Bote & $\mathrm{Zac}$ & Carrillo-González y \\
\hline Jatropha dioica & $\mathrm{Zn}$ & El Bote & $\mathrm{Zac}$ & González-Chávez 2006 \\
\hline Eichhornia crassipes & $\mathrm{Cr}, \mathrm{Mn}, \mathrm{Cd}$ & Xochimilco & $\mathrm{CdMx}$ & Carrión et al. 2012 \\
\hline Gnaphalium chartaceum & $\mathrm{Cu}, \mathrm{Mn}, \mathrm{Zn}, \mathrm{Pb}$ & Guerrero & Gro & \multirow[t]{3}{*}{ Cortés-Jiménez et al. 2013} \\
\hline Wigandia urens & $\mathrm{Zn}$ & Guerrero & Gro & \\
\hline Senecio salignus & $\mathrm{Zn}$ & Guerrero & Gro & \\
\hline Ambrosia artemisifolia & $\mathrm{Zn}$ & Villa de la Paz & SLP & \multirow[t]{4}{*}{ Franco-Hernández et al. 2010} \\
\hline Flaveria angustifolia & $\mathrm{As}, \mathrm{Cu}, \mathrm{Pb}, \mathrm{Zn}$ & Villa de la Paz & SLP & \\
\hline Flaveria trinervia & $\mathrm{As}, \mathrm{Cu}, \mathrm{Pb}, \mathrm{Zn}$ & Villa de la Paz & SLP & \\
\hline Simsia amplexicaulis & $\mathrm{Cu}$ & Villa de la Paz & SLP & \\
\hline Solanum corymbosum & $\mathrm{Cu}$ & Pachuca & Hgo & \multirow[t]{5}{*}{ Hernández-Acosta et al. 2009} \\
\hline Brickelia veronicifolia & $\mathrm{Pb}, \mathrm{Zn}$ & Pachuca & Hgo & \\
\hline Atriplex suberecta & $\mathrm{Cd}$ & Pachuca & Hgo & \\
\hline Cynodon dactylon & $\mathrm{Mn}$ & Pachuca & Hgo & \\
\hline Bouteloua curtipendula & $\mathrm{Ni}$ & Pachuca & Hgo & \\
\hline Amaranthus hybridus & $\mathrm{Pb}$ & Francisco I. Madero & $\mathrm{Zac}$ & \multirow[t]{3}{*}{ Salas-Luevano et al. 2009} \\
\hline Buddleja scordioides & $\mathrm{Pb}$ & Francisco I. Madero & $\mathrm{Zac}$ & \\
\hline Cerdia congestiflora & $\mathrm{Pb}$ & Francisco I. Madero & $\mathrm{Zac}$ & \\
\hline Nicotiana glauca & $\mathrm{As}, \mathrm{Cd}, \mathrm{Pb}, \mathrm{Zn}$ & Cadereyta de Montes & Qro & Santos-Jallath et al. 2012 \\
\hline
\end{tabular}

Abreviatura de los estados: Hidalgo (Hgo), San Luis Potosí (SLP), Zacatecas (Zac), Ciudad de México (CdMx), Guerrero (Gro), Querétaro (Qro). Símbolo químico de los metales: arsénico (As), plomo (Pb), cadmio (Cd), cromo (Cr), manganeso $(\mathrm{Mn})$, zinc $(\mathrm{Zn})$, cobre $(\mathrm{Cu})$, níquel $(\mathrm{Ni})$. 
remediación de metales en humedales artificiales (Carranza-Álvarez et al. 2008, Mauricio-Gutiérrez et al. 2010), por su capacidad de adaptación a ambientes contaminados, y su amplia distribución.

Una especie destacada por su potencial para la fitorremediación de metales es Jatropha dioica, la cual ha mostrado un FBC de 195.0, 6.5, 1.7 y 18.8 para los metales $\mathrm{Zn}, \mathrm{Cu}, \mathrm{Pb}$ y $\mathrm{Cd}$ respectivamente (Carrillo-González y González-Chávez 2006). Esta especie resulta atractiva por su potencial para ser utilizada en la producción de biocombustibles (Chandra-Pandey et al. 2012, Fresnedo-Ramírez y Orozco-Ramírez 2012).

Otra especie con potencial para ser utilizada en la fitorremediación es Amaranthus hybridus, debido a su amplia distribución y su rápido crecimiento (Puschenreiter et al. 2002). En México se ha evaluado la capacidad del género Amaranthus para acumular $\mathrm{Pb}$ y $\mathrm{Cd}$ al ser inoculado con hongos micorrízicos del género Glomus. En dicho estudio se observó una acumulación de 180 y $11 \mathrm{mg} / \mathrm{kg}$, respectivamente. Esta cantidad fue significativamente mayor que lo acumulado por los tratamientos sin inocular (OrtizCano et al. 2009).

\section{RIZOBACTERIAS PROMOTORAS DE CRECIMIENTO VEGETAL Y SU PAPEL EN TRATAMIENTOS DE FITORREMEDIACIÓN}

Algunas desventajas de la mayoría de las especies vegetales acumuladoras de metales es que son de lento crecimiento y producen poca biomasa (de Bashan et al. 2012), por ello se han buscado estrategias para incrementar su rendimiento en la fitoextracción de metales. Una manera de mejorar el proceso es mediante la inoculación con microorganismos del suelo (Glick 2003, Lebeau et al. 2008, Rajkumar et al. 2009).

Las principales características que se buscan en los microorganismos a utilizar en los tratamientos de fitorremediación es que cuenten con propiedades de promoción de crecimiento vegetal, además de la capacidad de movilizar metales (fitoextracción) o inmovilizarlos en las estructuras celulares (fitoestabilización) (Ma et al. 2009, Rajkumar et al. 2012).

Las bacterias logran estimular el crecimiento de la planta mediante varios mecanismos, como son la producción de fitohormonas para reducir los niveles de etileno (ACC-desaminasa) e incrementar el desarrollo radicular (ácido indolacético), o al mejorar el estatus nutricional de la planta a través de la fijación biológica de nitrógeno (Rajkumar y Freitas 2008,
Navarro-Noya et al. 2012, Rojas-Tapia et al. 2012). En relación con la capacidad de movilizar metales, ésta se puede lograr mediante la producción de ácidos orgánicos y de sideróforos (Rajkumar et al. 2012).

Por otro lado, los hongos micorrízicos al unirse simbióticamente con el sistema radicular de las plantas ocasionan un crecimiento en dicho sistema, lo que mejora principalmente la asimilación de fosfato (Gadd 2010). Asimismo, los metales pesados pueden ser inmovilizados mediante la secreción de glicoproteínas o ser adsorbidos en las paredes celulares fúngicas, reduciendo así su efecto tóxico para las plantas (Kirchman 2012). En la siguiente sección se describen brevemente los mecanismos mencionados.

\section{Ácido indolacético}

El ácido indolacético (AIA) es una de las fitohormonas más estudiadas. Es producida por rizobacterias y se ha demostrado que tiene un papel importante en el crecimiento de plantas en sitios contaminados con metales pesados al promover directamente la elongación y división celular o al alterar la expresión de algunos genes (Ma et al. 2009, de Bashan et al. 2012, Rajkumar et al. 2012).

Algunos géneros bacterianos productores de AIA como Burkholderia sp., Pseudomonas fluorescens y Microbacterium sp., al ser inoculados en mostaza india, tomate y maíz ocasionaron un incremento en la altura de la planta y en la concentración de $\mathrm{Pb}$ y Cd en la raíz y el tallo (Jiang et al. 2008, Sheng et al. 2008).

\section{1-Amino-ciclopropano-1-carboxilato desaminasa}

El etileno es una fitohormona que se encarga de modular el crecimiento y el metabolismo celular de las plantas. Puede producirse en respuesta a la exposición a una gran variedad de tipos de estrés (bióticos y abióticos) ocasionando generalmente una reducción en el crecimiento de la planta (Burd et al. 2000). Es sabido que la exposición a altas concentraciones de metales pesados puede inducir la sobreproducción de esta enzima, lo que afecta el crecimiento radicular y por consiguiente el desarrollo de la planta.

En este sentido algunas rizobacterias son capaces de reducir el efecto negativo causado por el etileno, mediante la producción de la enzima 1-aminociclopropano-1-carboxilato desaminasa (Glick 2003). Dicha enzima se encarga de hidrolizar la molécula precursora del etileno en $\alpha$ cetobutirato y amonio. El 1-amino-ciclopropano-1-carboxilato (ACC) es exudado por las raíces de las plantas y metabolizado en la rizósfera por la actividad de la ACC desaminasa 
bacteriana. Las bacterias con esta capacidad son utilizadas en los tratamientos de fitorremediación como inoculantes microbianos (Grichko et al. 2000, Arshad et al. 2007).

\section{FIJACIÓN BIOLÓGICA DE NITRÓGENO}

Es frecuente que los suelos contaminados con metales pesados presenten un bajo estatus nutrimental (Navarro-Noya et al. 2012). En este sentido, una opción para incrementar el contenido de nitrógeno disponible en el suelo es a través de la fijación biológica de $\mathrm{N}_{2}$ atmosférico. Dicha actividad puede ser llevada a cabo por bacterias en simbiosis estricta con leguminosas o de manera libre.

Entre los géneros de bacterias simbióticas fijadoras de $\mathrm{N}_{2}$ se encuentran: Rhizobium, Bradyrhizobium y Mesorhizobium. Mientras que entre los géneros de vida libre destacan Azospirillum, Herbaspirillum y Frankia (Gamalero et al. 2009).

Aunque se sabe que altas concentraciones de metales pesados como $\mathrm{Al}, \mathrm{Cr}$ y Cd pueden inhibir la fijación biológica de nitrógeno (Bishnoi et al. 1993, Balestrasse et al. 2003), también se ha reportado la formación de nódulos funcionales por poblaciones rizobiales adaptadas a altas concentraciones de metales pesados (Purchase et al. 1997, Carrasco et al. 2005).

Arora et al. (2010) reportan un efecto inhibitorio en la actividad de la nitrogenasa de cepas de Sinorhizobium y Bradyrhizobium debido a altas concentraciones de $\mathrm{Al}$ en condiciones in vitro. Sin embargo, en el caso de Fe y Mo reportan un aumento en la actividad de esta enzima, el cual puede deberse a que la nitrogenasa posee un átomo de Mo en su centro activo, además de centros de Fe y $\mathrm{S}$, necesarios para la reducción del nitrógeno atmosférico.

\section{SIDERÓFOROS}

Los sideróforos son compuestos de bajo peso molecular (1000 Da) que actúan como acarreadores de $\mathrm{Fe}^{+3}$ a través de las membranas de diferentes microorganismos. Los sideróforos se pueden agrupar de acuerdo con sus características de unión con el $\mathrm{Fe}^{+3}$ en tres clases: hidroxamatos, catecolatos y alfahidroxicarboxilatos (Kirchman 2012).

Los sideróforos aparte de su capacidad de unirse al $\mathrm{Fe}$, también pueden formar complejos con otros metales como $\mathrm{Al}, \mathrm{Cd}, \mathrm{Cu}, \mathrm{Pb}$ y $\mathrm{Zn}$, lo que ocasiona un incremento en la solubilidad de estos metales en el suelo (Rajkumar et al. 2010). Adicionalmente, la presencia de dichos metales estimula la producción de sideróforos por parte de las rizobacterias (Ma et al. 2009), siempre y cuando las concentraciones de metales en el medio no sobrepasen el límite de tolerancia de las bacterias (Kraemer et al. 2002, Naik y Dubey 2011).

\section{SOLUBILIZACIÓN DE FOSFATOS}

Algunas bacterias rizosféricas tienen la capacidad de solubilizar fosfatos debido a la producción de ácidos orgánicos de bajo peso molecular (Gupta et al. 2002), entre los cuales están los ácidos fórmico, acético, succínico, tartárico y oxálico (Li et al. 2010). Se ha demostrado que los ácidos orgánicos excretados por las bacterias rizosféricas también tienen efecto en la movilización de metales como el $\mathrm{Pb}, \mathrm{Ni}, \mathrm{Cu}$, Cd y Zn (Fasim et al. 2002, Arwidsson et al. 2010, Li et al. 2010). Esto se asume debido a la excreción de los ácidos orgánicos al suelo que ocasiona una disminución del pH (Sheng y Shia 2006), que a su vez libera los cationes metálicos al medio.

\section{ESTUDIOS DE FITORREMEDIACIÓN EN MÉXICO}

En México las investigaciones se han enfocado al proceso de fitoextracción partiendo de la utilización de plantas nativas acumuladoras de metales y del conocimiento de la diversidad microbiana de cada zona particular para obtener aislados que promuevan el crecimiento vegetal y ayuden al proceso de fitorremediación al ser inoculados en las plantas.

El estudio de los humedales como tecnología para el tratamiento de metales pesados cuenta con muchos avances científicos. Específicamente en el estudio de comunidades microbianas asociadas a la rizósfera de Scirpus americanus donde se han identificado géneros como Agrobacterium, Arthrobacter, Microbacterium, Curtobacterium, Rhodococcus, Xanthomonas y Pseudomonas con la capacidad de reducir Cr (IV) a Cr (III) (Mauricio-Gutiérrez et al. 2010).

Asimismo, otra especie que ha resultado eficiente en la eliminación de $\mathrm{Cr}$ de efluentes contaminados es Typha sp., que al ser utilizada en combinación con Scirpus americanus logró reducir hasta $99 \%$ de $\mathrm{Cr}$ en efluentes de una curtiduría, además de disminuir $99 \%$ de la concentración de sulfuros y $92 \%$ de sulfatos en el agua (Pacheco-Aguilar et al. 2008). Dicha reducción en compuestos de $\mathrm{S}$ se atribuye a la comunidad de bacterias sulfuro-oxidantes presentes 
en el estanque, donde algunos géneros como Acinetobacter, Alcaligenes, Ochrobactrum y Pseudomonas presentaron la capacidad de oxidar compuestos reducidos de azufre.

Debido a que los suelos contaminados con jales mineros son a menudo pobres en nutrientes, se busca el uso de microorganismos que puedan aportar los elementos necesarios para el crecimiento de las plantas y que a su vez tengan la capacidad de crecer en concentraciones altas de metales pesados.

En este sentido se han estudiado y caracterizado bacterias fijadoras de $\mathrm{N}_{2}$, tanto de vida libre como simbióticas provenientes de sitios contaminados con metales pesados. Especies como Azospirillum lipoferum, Bradyrhizobium japonicum y Paenibacillus sp. con tolerancia a $\mathrm{Cd}, \mathrm{Cr}$ y $\mathrm{Zn}$, fueron aisladas de la rizósfera de plantas creciendo en jales mineros en Zacatecas. Estas especies mostraron una alta actividad de nitrogenasa en presencia de metales (Navarro-Noya et al. 2012).

Otras especies de plantas nativas que se han utilizado para fitorremediación son Eleocharis macrostachya, Schoenoplectus americanus y Baccharis salicifolia (Alarcón-Herrera et al. 2012), que se adaptaron a un sustrato contaminado con As, siendo $S$. americanus la que presentó un mayor desarrollo comparado con las demás. Los valores de FT (1.73) y FBC (59.74) obtenidos para esta especie indican su potencial para ser utilizada con fines de fitorremediación.

Sin embargo, el tratamiento de fitorremediación no termina cuando el metal es acumulado en el tejido de las plantas ya que se necesita una disposición final. Es por eso que se busca generar tratamientos integrales que incluyan opciones para el manejo de los residuos. Al respecto Nuñez-Lopez et al. (2009), establecieron un sistema para la recuperación de plomo a partir del tejido de Eichhornia crassipes, el cual consistió en dos etapas: una lixiviación química con oxalato de amonio y una segunda etapa con una recuperación electroquímica del plomo a partir del lixiviado, con lo que se obtuvo una eficiencia del $95 \%$.

\section{CONCLUSIONES Y PERSPECTIVAS}

La contaminación por metales pesados en México es un tema que ha recibido poca cobertura por parte de las autoridades, no obstante la magnitud y los focos de alerta derivados de los estudios de las zonas de riesgo. Sin embargo, recientemente se ha incrementado el número de reportes científicos donde se ilustra la relevancia de la aplicación de la biotecnología en los estudios de biorremediación. Específicamente, la fitorremediación asistida por microorganismos ha surgido como una alternativa para mejorar la eficacia en los tratamientos de desintoxicación de sistemas contaminados por metales pesados. En México, se han identificado especies vegetales con capacidad de absorción de metales pesados y capaces de establecer relaciones mutualistas con microorganismos, mejorando así su capacidad de fitorremediación. Algunos de los géneros vegetales más estudiados son Thypa, Crassipes, Acaccia, Amaranthus, Prosopis y Helianthus, por otro lado entre las bacterias más estudiadas están los géneros Rhizobium, Agrobacterium, Arthrobacter, Microbacterium, Curtobacterium, Rhodococcus, Xanthomonas y Pseudomonas, así como hongos del género Glomus. La optimización funcional de los sistemas planta-microorganismo a través de estudios de ingeniería genética ayudaría a disminuir los tiempos de tratamiento y a reducir los riesgos a la salud en grupos vulnerables.

\section{AGRADECIMIENTOS}

Los autores agradecen al Consejo Nacional de Ciencia y Tecnología (CONACyT) por el apoyo brindado para la realización de esta revisión a través del proyecto "La fitorremediación como alternativa para atenuar la contaminación por metales pesados y compuestos organoclorados en Zacatecas" (FOINS 214108).

\section{REFERENCIAS}

Alarcón-Herrera M.T., Olmos-Márquez M.A., VallesAragon C., Llorens E. y Martín-Domínguez I.R. (2012). Assesments of plants for phytoremediation of arsenic-contaminated water and soil. Eur. Chem. Bull. 2, 121-125.

Ali H., Khan E. y Sjad M.A. (2013). Phytoremediation of heavy metals-concepts and applications. Chemosphere 91, 869-881.

DOI: 10.1016/j.chemosphere.2013.01.075

Alloway B.J. (2013). Heavy metals in soils, trace metals and metalloids in soils and their bioavailability. 3ra edición. Springer, Reading, Reino Unido, 587 pp. DOI: 10.1007/978-94-007-4470-7

Armienta M.A., Ongley L.K., Rodríguez R., Cruz O., Mango H. y Villaseñor G. (2008). Arsenic distribution in mesquite (Prosopis laevigata) and huizache (Acacia farnesiana) in the Zimapán mining area, México. Geochem. Explor. Environ. Anal. 8, 191-197.

DOI: $10.1144 / 1467-7873 / 07-144$ 
Armienta M.A., Rodríguez R. y Cruz O. (1997a). Arsenic content in hair of people exposed to natural arsenic polluted groundwater at Zimapán, Mexico. Bull. Environ. Contam. Toxicol. 59, 583-589.

DOI: $10.1007 / \mathrm{s} 001289900519$

Armienta M.A., Rodríguez R., Aguayo A., Ceniceros N., Villaseñor G. y Cruz O. (1997b). Arsenic contamination of groundwater at Zimapán, Mexico. Hydrogeol. J. 5, 39-46. DOI: 10.1007/s100400050111

Armienta M.A., Rodríguez R., Ceniceros N., Juárez F. y Cruz O. (1996). Distribution, origin and fate of chromium in soils in Guanajuato, Mexico. Environ. Pollut. 91, 391-397. DOI: 10.1016/0269-7491(95)00040-2

Armienta M.A., Villaseñor G., Rodríguez R., Ongley L.K. y Mango H. (2000). The role of arsenic-bearing rocks in groundwater pollution at Zimapán Valley, México. Environ. Geol. 40, 571-581.

DOI: $10.1007 / \mathrm{s} 002540000220$

Arora N.K., Khare E., Singh S. y Maheshwari D.K. (2010). Effect of Al and heavy metals on enzyme of nitrogen metabolism of fast and slow growing rhizobia under explanta conditions. World J. Microbiol. Biotechnol. 26, 811-816. DOI: 10.1007/s11274-009-0237-6

Arshad M., Saleem M. y Hussain S. (2007). Perspectives of bacterial ACC deaminase in phytoremediation. Trends Biotechnol. 25, 356-362.

DOI: 10.1016/j.tibtech.2007.05.005

Arwidsson Z., Johansson E., von Kronhelm T., Allard B. y van Hees P. (2010). Remediation of metal contaminated soil by organic metabolites from fungi I-production of organic acids. Water, Air, Soil Pollut. 205, 215-226. DOI: $10.1007 / \mathrm{s} 11270-009-0067-\mathrm{z}$

ATSDR (2007). ToxFAQs for Lead. Agency for toxic substances and disease registry [en línea] http://www. atsdr.cdc.gov/toxfaqs/tfacts13.pdf 18/09/2016.

ATSDR (2012). ToxFAQs for Chromium. Agency for toxic substances and disease registry [en línea]. http://www. atsdr.cdc.gov/toxfaqs/tfacts7.pdf 18/09/2016.

Baker A.J.M. (1981). Accumulators and excluders -strategies in the response of plants to heavy metals. J. Plant Nutr. 3, 643-654. DOI: 10.1080/01904168109362867

Balestrasse K.B., Benavides M.P., Gallego S.M. y Tomaro M.L. (2003). Effect of cadmium stress on nitrogen metabolism in nodules and roots of soybean plants. Funct. Plant. Biol. 30, 57-64. DOI: 10.1071/FP02074

Bishnoi N.R., Chugh L.K. y Sawhney S.K. (1993). Effect of chromium on photosynthesis, respiration and nitrogen fixation in pea (Pisum sativum L.) seedlings. J. Plant Physiol. 142, 25-30.

DOI: $10.1016 / \mathrm{S} 0176-1617(11) 80102-1$

Brookes P.C. (1995). The use of microbial parameters in monitoring soil pollution by heavy metals. Biol. Fertil. Soils. 19, 269-279. DOI: 10.1007/BF00336094
Burd G.I., Dixon D.G. y Glick B.R. (2000). Plant growth promoting bacteria that decrease heavy metal toxicity in plants. Can. J. Microbiol. 46, 237-245.

DOI: $10.1139 /$ w99-143

Carranza-Álvarez C., Alonso-Castro A.J., Alfaro de la Torre M.C. y García de la Cruz R.F. (2008). Accumulation and distribution of heavy metals in Scirpus americanus and Typha latifolia from an artificial lagoon in San Luis Potosí México. Water, Air, Soil Pollut. 188, 297-309.

DOI: $10.1007 / \mathrm{s} 11270-007-9545-3$

Carrasco J.A., Armario P., Pajuelo E., Burgos A., Caviedes M.A., López R., Chamber M.A. y Palomares A.J. (2005). Isolation and characterisation of symbiotically effective Rhizobium resistant to arsenic and heavy metals after the toxic spill at the Aznalcóllar pyrite mine. Soil Biol. Biochem. 37, 1131-1140.

DOI: 10.1016/j.soilbio.2004.11.015

Carrillo-González R. y González-Chávez M.C.A. (2006). Metal accumulation in wild plants surrounding mining wastes. Environ. Pollut. 144, 84-92.

DOI: 10.1016/j.envpol.2006.01.006

Carrión C., Ponce de León C., Cram S., Sommer I., Hernández M. y Vanegas C. (2012). Aprovechamiento potencial del lirio acuático (Eichhornia crassipes) en Xochimilco para fitorremediación de metales. Agrociencia 46, 609-620.

Castro-Díaz J. (2011). Informe sobre el mercado de mercurio en México. Comisión para la Cooperación Ambiental (CCA) de América del Norte [en línea]. http:// www3.cec.org/islandora/es/item/10100-mexicanmercury-market-report-es.pdf 18/09/2018.

Castro-Larragoitia J., Kramar U. y Puchelt H. (1997). 200 years of mining activities at La Paz/San Luis Potosi/ Mexico-Consequences for environment and geochemical exploration. J. Geochem. Explor. 58, 81-91.

DOI: $10.1016 / \mathrm{S} 0375-6742(96) 00054-4$

Chandra-Pandey V., Singh K., Shankar-Singh J., Kumar A., Singh B. y Singh R.P. (2012). Jatropha curcas: A potential biofuel plant for sustainable environmental development. Renew. Sust. Energ. Rev. 16, 2870-2883. DOI: 10.1016/j.rser.2012.02.004

Cortés-Jiménez E.V., Mugica-Álvarez V., GonzálezChávez M.C., Carrillo-González R., Martínez-Gordillo M. y Vaca-Mier M. (2013). Natural revegetation of alkaline tailing heaps at Taxco, Guerrero, Mexico. Int. J. Phytoremediat. 15, 127-141.

DOI: $10.1080 / 15226514.2012 .683208$

Costilla-Salazar R., Trejo-Acevedo A., Rocha-Amador D., Gaspar-Ramírez O., Díaz-Barriga F. y PérezMaldonado I.N. (2010). Assessment of polychlorinated biphenyls and mercury levels in soil and biological samples from San Felipe, Nuevo Mercurio, Zacatecas, 
Mexico. Bull. Environ. Contam. Toxicol. 86, 212-216. DOI: $10.1007 / \mathrm{s} 00128-010-0165-\mathrm{z}$

de la Rosa D.A., Volke-Sepulveda T., Solorzano G., Green C., Tordon R. y Beauchamp S. (2004). Survey of atmospheric total gaseous mercury in Mexico. Atmos. Environ. 38, 4839-4846.

DOI: 10.1016/j.atmosenv.2004.06.013

de-Bashan L.E., Hernandez J.P. y Bashan Y. (2012). The potential contribution of plant growth-promoting bacteria to reduce environmental degradation - A comprehensive evaluation. Appl. Soil Ecol. 61, 171189. DOI: $10.1016 /$ j.apsoil.2011.09.003

Duffus J.H. (2002). Heavy metals - A meaningless term? (IUPAC Technical Report). Pure Appl. Chem. 74, 793807. DOI: $10.1351 /$ pac200274050793

Espinosa E., Armienta M.A., Cruz O., Aguayo A. y Ceniceros N. (2009). Geochemical distribution of arsenic, cadmium, lead and zinc in river sediments affected by tailings in Zimapan, a historical polymetalic mining zone of Mexico. Environ. Geol. 58, 1467-1477.

DOI: $10.1007 / \mathrm{s} 00254-008-1649-6$

Fasim F., Ahmed N., Parsons R. y Gadd G.M. (2002). Solubilization of zinc salts by a bacterium isolated from the air environment of a tannery. FEMS Microbiol. Lett. 213, 1-6.

DOI: 10.1111/j.1574-6968.2002.tb11277.x

Fernández-Bremauntz A., Yarto-Ramírez M. y CastroDíaz J. (2004). Las sustancias tóxicas persistentes. Secretaria de Medio Ambiente y Recursos Naturales e Instituto Nacional de Ecología. Ciudad de México, México, 260 pp.

Flores-Ramírez R., Rico-Escobar E., Núñez-Monreal J.E., García-Nieto E., Carrizales L., Lizaliturri-Hernández y Díaz-Barriga F. (2012). Exposición infantil al plomo en sitios contaminados. Salud Pública México 54, 383-392. DOI: 10.1590/S0036-36342012000400008

Franco-Hernández M.O., Vásquez-Murrieta M.S., PatiñoSiciliano A. y Dendooven L. (2010). Heavy metal concentration in plants growing on mine tailings in Central Mexico. Bioresour. Technol. 101, 3864-3869. DOI: $10.1016 /$ j.biortech.2010.01.013

Fresnedo-Ramírez J. y Orozco-Ramírez Q. (2012). Diversity and distribution of genus Jatropha in Mexico. Genet. Resour. Crop. Evol. 60, 1087-1104. DOI: $10.1007 / \mathrm{s} 10722-012-9906-7$

Gadd G.M. (2010). Metals, minerals and microbes: geomicrobiology and bioremediation. Microbiology 158, 609-643. DOI: 10.1099/mic.0.037143-0

Gamalero E., Lingua G., Berta G. y Glick B.R. (2009). Beneficial role of plant growth promoting bacteria and arbuscular mycorrhizal fungi on plant responses to heavy metal stress. Can. J. Microbiol. 55, 501-514. DOI: 10.1139/W09-010
García-Hernández J., Cadena-Cárdenas L., BetancourtLozano M., García de la Parra L.M., García-Rico L. y Márquez-Farías F. (2007). Total mercury content found in edible tissues of top predator fish from the Gulf of California, Mexico. Toxicol. Environ. Chem. 89, 507522. DOI: $10.1080 / 02772240601165594$

Glick B.R. (2003). Bacterial ACC-deaminase and the alleviation of plant stress. Adv. Appl. Microbiol. 56, 291-312. DOI: 10.1016/S0065-2164(04)56009-4

González-Dávila O., Gómez-Bernal J.M. y Ruíz-Huerta E.A. (2012). Plants and soil contamination with heavy metals in agricultural areas of Guadalupe, Zacatecas, Mexico. En: Environmental contamination. (J. K. Srivastava, Ed.). InTech. Rijeka, Croacia, pp. 37-50. DOI: $10.5772 / 31062$

González-Valdez E., González-Reyes E., Bedolla-Cedeño C., Arrollo-Ordaz E.L. y Manzanares-Acuña E. (2008). Blood lead levels and risk factors for lead poisoning in mexican children. Rev. Fac. Ing.-Univ. Ant. 43, 114-119.

Gratao P.L., Prasad M.N.V., Cardoso P.F., Lea P.J. y Azevedo R.A. (2005). Phytoremediation: green technology for the clean-up of toxic metals in the environment. Braz. J. Plant Physiol. 17, 53-64.

DOI: $10.1590 / \mathrm{S} 1677-04202005000100005$

Grichko V.P., Filby B. y Glick B.R. (2000). Increased ability of transgenic plants expressing the bacterial enzyme ACC deaminase to accumulate $\mathrm{Cd}, \mathrm{Co}, \mathrm{Cu}$, $\mathrm{Ni}, \mathrm{Pb}$, and Zn. J. Biotechnol. 81, 45-53.

DOI: $10.1016 / \mathrm{S} 0168-1656(00) 00270-4$

Guentzel J.L., Portilla E., Keith K.M. y Keith E.O. (2007). Mercury transport and bioacumulation in riverbank communities of the Alvarado lagoon system, Veracruz state Mexico. Sci. Total Environ. 388, 316-324.

DOI: $10.1016 /$ j.scitotenv.2007.07.060

Gupta A., Meyer J.M. y Goel R. (2002). Development of heavy metal-resistant mutants of phosphate solubilizing Pseudomonas sp. NBRI 4014 and their characterization. Curr. Microbiol. 45, 323-327.

DOI: $10.1007 / \mathrm{s} 00284-002-3762-1$

Hernández-Acosta E., Mondragón-Romero E., CristóbalAcevedo D., Rubiños-Panta J.E. y Robledo-Santoyo E. (2009). Vegetación, residuos de mina y elementos potencialmente tóxicos de un jal de Pachuca Hidalgo, México. Rev. Chapingo Ser. Cie. 15, 109-114.

Hernández-Silva G., García-Martínez R., Solís-Valdez S., Martínez-Trinidad S., Mercado-Sotelo I., RamírezIslas M., Scharek P. y Solorio-Munguía G. (2012). Presencia de Hg total en una relación planta-sueloatmósfera al sur de la Sierra Gorda Querétaro, México. Rev. Esp. Cienc. Quím. Biol. 15, 5-15.

Iskander F.Y., Vega-Carrillo H.R. y Manzanares-Acuña E. (1994). Determination of mercury and other elements 
in la Zacatecana Dam sediment in Mexico. Sci. Total Environ. 148, 45-48.

DOI: 10.1016/0048-9697(94)90372-7

Jiang C., Sheng X., Qian M. y Wang Q. (2008). Isolation and characterization of a heavy metal-resistant Burkholderia sp. from heavy metal-contaminated paddy field soil and its potential in promoting plant growth and heavy metal accumulation in metal-polluted soil. Chemosphere 72, 157-164.

DOI: 10.1016/j.chemosphere.2008.02.006

Kirchman D.L. (2012). Processes in microbial ecology. 1era edición Oxford University Press Inc. Nueva York, EUA, 312 pp.

DOI: 10.1093/acprof:oso/9780199586936.001.0001

Kraemer S.T., Xu J., Raymond K.N. y Sposito G. (2002). Adsorption of $\mathrm{Pb}$ (II) and $\mathrm{Eu}$ (III) by oxide minerals in the presence of natural and synthetic hydroxamate siderophores. Environ. Sci. Technol. 36, 1287-1291. DOI: $10.1021 / \mathrm{es} 010182 \mathrm{c}$

Lebeau T., Braud A. y Jezequel K. (2008). Performance of bioaugmentation-assited phytoextraction applied to metal contaminated soils: a review. Environ. Pollut, 153, 497-522. DOI: 10.1016/j.envpol.2007.09.015

Li P., Feng X.B., Shang L.H., Qiu G., Meng B., Zhang H., Guo Y. y Liang P. (2011). Human co-exposure to mercury vapor and methylmercury in artisanal mercury mining areas, Guizhou, China. Ecotoxicol. Environ. Saf. 74, 473-479. DOI: 10.1016/j.ecoenv.2010.10.030

Li W.C. y Tse H.F. (2015). Health risk and significance of mercury in the environment. Environ. Sci. Pollut. Res. 22, 192-201. DOI: 10.1007/s11356-014-3544-x

Li W.C., Ye Z.H. y Wong M.H. (2010). Metal mobilization and production of short-chain organic acids by rhizosphere bacteria associated with a $\mathrm{Cd} / \mathrm{Zn}$ hyperaccumulating plant, Sedum alfredii. Plant Soil. 326, 453-467. DOI: 10.1007/s11104-009-0025-y

Ma Y., Rakjumar M. y Freitas H. (2009). Isolation and characterization of Ni mobilizing PGPB from serpentine soils and their potential in promoting plant growth and Ni accumulation by Brassica spp. Chemosphere 75, 719-725. DOI: 10.1016/j.chemosphere.2009.01.056

Martínez-Trinidad S., Hernández-Silva G., MartínezReyes J., Solorio-Munguía G., Solís-Valdez S., Ramírez-Islas M.E. y García-Martínez R. (2013). Total Mercury in terrestrial systems (airl-soil-plant-water) at the mining región of San Joaquín, Querétaro, México. Geo. Inter. 52, 43-58.

DOI: 10.1016/S0016-7169(13)71461-2

Mauricio-Gutiérrez A., Peña-Cabriales J.J. y MaldonadoVega M. (2010). Isolation and characterization of hexavalent Chromium-reducing rhizospheric bacteria from a wetland. Int. J. Phytoremediat. 12, 317-334. DOI: $10.1080 / 15226510902968118$
Meharg A.A. y Zaho F.J. (2012). Arsenic and Rice. Springer Science+Business Media B.V. Dordrecht, Holanda, 166 pp. DOI: 10.1007/978-94-007-2947-6

Meza-Figueroa D., Maier R.M., de la O-Villanueva M., Gómez-Álvarez A., Moreno-Zazueta A., Rivera J., Campillo A., Grandlic C.J., Anaya R. y Palafox-Reyes J. (2009). The impact of unconfined mine tailings in residential areas from a mining town in a semi-arid environment: Nacozari, Sonora, Mexico. Chemosphere 77, 140-147.

DOI: 10.1016/j.chemosphere.2009.04.068

Mireles F., Dávila J.I., Pinedo J.L., Reyes E., Speakman R.J. y Glascock M.D. (2012). Assessing urban soil pollution in the cities of Zacatecas and Guadalupe, Mexico by instrumental neutron activation analysis. Microchem. J. 103, 158-164.

DOI: 10.1016/j.microc.2012.02.009

Mithöfer A., Shulze B. y Boland-Whilheim. (2004). Biotic and heavy metal stress response in plants: evidence for common signals. FEBS Lett. 566, 1-5.

DOI: $10.1016 /$ j.febslet.2004.04.011

Naik M.M. y Dubey S.M. (2011). Lead enhanced siderophore production and alteration in cell morphology in a Pb-resistant Pseudomonas aureginosa strain 4EA. Curr. Microbiol. 62, 409-414.

DOI: $10.1007 / \mathrm{s} 00284-010-9722-2$

Navarro-Noya Y.E., Hernández-Mendoza E., MoralesJiménez J., Jan-Roblero J., Martínez-Romero E. y Hernández-Rodríguez C. (2012). Isolation and characterization of nitrogen fixing heterotrophic bacteria from the rhizosphere of Pioneer plants growing on mine tailings. Appl. Soil. Ecol. 62, 52-60.

DOI: 10.1016/j.apsoil.2012.07.011

Nuñez-López R.A., Meas-Vong Y., Ortega-Borges R. y Olguín E.J. (2009). Rizofiltración, Lixiviación y Electro-Recuperación: Una Propuesta Integral para el Tratamiento de Aguas Contaminadas con Plomo a Partir del Lirio Acuático (Eichornia crassipes). Ciencia@UAQ.2, 17-31.

Olguín E. y Sánchez-Galván G. (2012). Heavy metal removal in phytofiltration and phycoremediation: the need to differentiate between bioadsorption and bioaccumulation. N. Biotechnol. 30, 3-8.

DOI: $10.1016 /$ j.nbt.2012.05.020

Ongley L.K., Sherman L., Armienta A., Concilio A. y Ferguson-Salinas C. (2007). Arsenic in the soils of Zimapan, Mexico. Environ. Pollut. 145, 793-799.

DOI: 10.1016/j.envpol.2006.05.014

Ortiz-Cano H.G., Trejo-Calzada R., Valdez-Cepeda R.D., Arreola-Ávila J.G., Flores-Hernández A. y López-Ariza B. (2009). Fitoextracción de plomo y cadmio en suelos contaminados usando quelite (Amaranthus hybridus L.) y micorrizas. Rev. Chap. Ser. Hort. 15, 161-168. 
Pacheco-Aguilar R., Peña-Cabriales J.J. y MaldonadoVega M. (2008). Identification and characterization of sulfur-oxidizing bacteria in an artificial wetland that treats wastewater from a tannery. Int. J. Phytoremediat. 10, 359-370. DOI: 10.1080/15226510802100390

Peralta-Videa J.R., López M.L., Narayan M., Saupe G. y Gardea-Torresdey J. (2009). The biochemistry of environmental heavy metal uptake by plants: Implications for the food chain. Int. J. Biochem. Cell Biol. 41, 1665-1677. DOI: 10.1016/j.biocel.2009.03.005

Pérez-Vázquez F.J., Flores-Ramírez R., Ochoa-Martínez A.C., Orta-García S.T., Hernandez-Castro B., Carrizalez-Yáñez L. y Pérez-Maldonado I.N. (2015). Concentrations of persistent organic pollutants (POPs) and heavy metals in soil from San Luis Potosí, México. Environ. Monit. Asses. 187, 4119.

DOI: $10.1007 / \mathrm{s} 10661-014-4119-5$.

Pilon-Smits E. (2005). Phytoremediation. Annu. Rev. Plant Biol. 56, 15-39.

DOI: 10.1146/annurev.arplant.56.032604.144214

Prasad M.N.V. y Freitas H. (2003). Metal hyperaccumulation in plants - Biodiversity prospecting for phytoremediation technology. Electron. J. Biotechnol. 6, 275-321. DOI: $10.2225 /$ vol6-issue3-fulltext-6

Prohaska T. y Stingeder G. (2005). Speciation of arsenic. En: Handbook of elemental speciation II - species in the environment, food, medicine and occupational health. (R. Cornelis, J. Caruso, Eds.). John Wiley and Sons Ltd, Chicester, Reino Unido, pp. 69-85.

DOI: 10.1002/0470856009

Purchase D., Miles R.J. y Young T.W.K. (1997). Cadmium uptake and nitrogen fixing ability in heavy-metalresistant laboratory and field strains of Rhizobium leguminosarum biovar trifolii. FEMS Microbiol. Ecol. 22, 85-93. DOI: 10.1111/j.1574-6941.1997.tb00359.x

Puschenreiter M., Stöger G., Lombi E., Horak O. y Wenzel W.W. (2002). Phytoextraction of heavy metal contaminated soils with Thlaspi goesingense and Amaranthus hybridus: Rhizosphere manipulation using EDTA and ammonium sulfate. J. Plant Nutr. Soil Sci. 164, 615-621. DOI: 10.1002/1522-2624(200112)164:6<615::AIDJPLN615>3.0.CO;2-6

Rajkumar M. y Freitas H. (2008). Influence of metal resistant-plant growth-promoting bacteria on the growth of Ricinus communis in soil contaminated with heavy metals. Chemosphere 71, 834-842.

DOI: 10.1016/j.chemosphere.2007.11.038

Rajkumar M., Ae N. y Freitas H. (2009). Endophytic bacteria and their potential to enhance heavy metal phytoextraction. Chemosphere 77, 153-160. DOI: 10.1016/j.chemosphere.2009.06.047

Rajkumar M., Ae N., Prasad M.N.V. y Freitas H. (2010). Potential of siderophore-producing bacteria for improving heavy metal phytoextraction. Trends. Biotechnol. 28, 142-149.

DOI: 10.1016/j.tibtech.2009.12.002

Rajkumar M., Sandhya S., Prasad M.N.V. y Freitas H. (2012). Perspectives of plant-associated microbes in heavy metal phytoremediation. Biotechnol. Adv. 30, 1562-1574. DOI: 10.1016/j.biotechadv.2012.04.011

Ramos-Arroyo Y.R. y Siebe-Grabach C.D. (2006). Estrategia para identificar jales con potencial de riesgo ambiental en un distrito minero: estudio de caso en el Distrito de Guanajuato, México. Rev. Mex. Cienc. Geol. 23, 54-74.

Rascio N. y Navari-Izzo F. (2011). Heavy metal hyperaccumalating plants: How and why do they do it? And what makes them so interesting? Plant Science 180, 169-181. DOI: 10.1016/j.plantsci.2010.08.016

Razo I., Carrizales L., Castro J., Díaz-Barriga F. y Monroy M. (2003). Arsenic and heavy metal pollution of soil, water and sediments in a semi-arid climate mining area in Mexico. Water, Air, Soil Pollut. 152, 129-152. DOI: 10.1023/B:WATE.0000015350.14520.c1

Ribeiro de Souza S.C., López de Andrade S.A., Anjos de Souza L. y Aparecida Schiavinato M. (2012). Lead tolerance and phytoremediation potential of Brazilian leguminous tree species at the seedling stage. J. Environ. Manage. 110, 299-307.

DOI: 10.1016/j.jenvman.2012.06.015

Robles-Camacho J. y Armienta M.A. (2000). Natural chromium contamination of groundwater at Leon Valley Mexico. J. Geochem. Explor. 68, 167-181. DOI: $10.1016 / \mathrm{S} 0375-6742(99) 00083-7$

Rojas-Tapia D.F., Bonilla R.R. y Dussán J. (2012). Effect of inoculation with plant growth-promoting Bacteria on growth and copper uptake by sunflowers. Water, Air, Soil Pollut. 223, 643-654.

DOI: $10.1007 / \mathrm{s} 11270-011-0889-3$

Romero F.M. y Gutiérrez-Ruiz M. (2010). Estudio comparativo de la peligrosidad de jales en dos zonas mineras localizadas en el sur y centro de México. Bol. Soc. Geol. Mex. 64, 43-53.

Romero F.M., Armienta M.A. y González-Hernández G. (2007). Solid phase control on the mobility of potentially toxic elements in an abandoned lead/zinc mine tailings impoundment, Taxco, Mexico. Appli. Geochemestry 22, 109-127. DOI: 10.1016/j.apgeochem.2006.07.017

Ruelas-Inzunza J., Páez-Osuna F., Ruiz-Fernández A.C. y Zamora-Arellano N. (2011). Health risk associated to dietary intake of mercury in selected Coastal Areas of Mexico. Bull. Environ. Contam. Toxicol. 86, 180-188. DOI: $10.1007 / \mathrm{s} 00128-011-0189-\mathrm{z}$

Salas-Luevano M.A., Manzanares-Acuña E., Letechipia de León C. y Vega-Carrillo H.R. (2009). Tolerant 
and hyperaccumulators autochthonous plant species from mine tailing disposal sites. Asian J. Exp. Sci. $23,27-32$.

Santos-Jallath J., Castro-Rodríguez A., Huezo-Casillas J. y Torres-Bustillos L. (2012). Arsenic and heavy metals in native plants at tailings impoundments in Queretaro, Mexico. Phys. Chem. Earth 37-39, 10-17. DOI: 10.1016/j.pce.2011.12.002

Santos-Santos E., Yarto-Ramírez M., Gavilán-García I., Castro-Díaz J., Gavilán-García A., Rosiles R., Suárez S. y López-Villegas T. (2006). Analysis of arsenic, lead and mercury in farming areas with mining contaminated soils at Zacatecas, Mexico. J. Mex. Chem. Soc. 50, 57-63.

Schützendübel A. y Polle A. (2002). Plant responses to abiotic stresses: heavy metal-induced oxidative stress and protection by mycorrhization. J. Exp. Bot. 53, 1351-1365. DOI: 10.1093/jexbot/53.372.1351

SEMARNAT (2003). Norma Oficial Mexicana NOM001-SEMARNAT 1996. Que establece los límites máximos permisibles de contaminantes en las descargas de aguas residuales en aguas y bienes nacionales. Secretaría de Medio Ambiente, Recursos Naturales y Pesca. Diario Oficial de la Federación. 23 abril 2003.

SEMARNAT (2007). Norma Oficial Mexicana. NOM147-SEMARNAT/SSA1-2004. Que establece los criterios para determinar las concentraciones de remediación de suelos contaminados por arsénico, bario, berilio, cadmio, cromo hexavalente, mercurio, níquel, plata, plomo, selenio, talio y/o vanadio. Secretaría de Medio Ambiente, Recursos Naturales y Pesca. Diario Oficial de la Federación. 2 de marzo de 2007.

Sheng X., Xia J., Jiang C., He L. y Qian M. (2008). Characterization of heavy metal-resistant endophytic bacteria from rape (Brassica napus) roots and their potential in promoting the growth and lead accumulation of rape. Environ. Pollut. 156, 1164-1170.

DOI: 10.1016/j.envpol.2008.04.007

Sheng X.F. y Xia J.J. (2006). Improvement of rape (Brassica napus) plant growth and cadmium uptake by cadmium-resistant bacteria. Chemosphere 64, 10361042. DOI: 10.1016/j.chemosphere.2006.01.051
Singh N. y Ma L.Q. (2006). Arsenic speciation, and arsenic and phosphate distribution in arsenic hyperaccumulator Pteris vittata L. and non-hyperaccumulator Pteris ensiformis. Environ. Pollut. 141, 238-246.

DOI: 10.1016/j.envpol.2005.08.050

Smeets K., Cuypers A., Lambrechts A., Semane B., Hoet P., Van Laere A. y Vangronsveld J. (2005). Induction of oxidative stress and antioxidative mechanisms in Phaseolus vulgaris after Cd application. Plant Physiol. Biochem. 43, 437-444.

DOI: 10.1016/j.plaphy.2005.03.007

Trejo-Acevedo A., Díaz-Barriga F., Carrizales L., Domínguez G., Costilla R., Ize-Lema I., Yarto-Ramírez M., Gavilán-García A., Mejía-Saavedra J.J. y Pérez-Maldonado I.N. (2009). Exposure assessment of persistent organic pollutants and metals in Mexican Children. Chemosphere 74, 974-980.

DOI: 10.1016/j.chemosphere.2008.10.030

UNEP (2013). Global mercury assessment 2013: Sources, emissions, releases and environmental transport. United Nations Environmental Programme Chemicals Branch, Ginebra, Suiza, 32 pp.

Velasco-González O.H., Echavarría-Almeida S., PérezLópez M.E. y Villanueva-Fierro I. (2001). Contenido de mercurio y arsénico en atún y sardinas enlatadas mexicanas. Rev. Int. Contam. Ambie. 17, 31-35.

Villanueva S. y Botello A. (1992). Metales pesados en la zona costera del Golfo de México y Caribe Mexicano: una revisión. Rev. Int. Contam. Ambie. 8, 47-61.

Volke-Sepulveda T., Velasco-Trejo J.A. y de la Rosa Pérez D.A. (2005). Suelos contaminados por metales y metaloides: muestreo y alternativas para su remediación. Secretaría de Medio Ambiente y Recursos Naturales. Instituto Nacional de Ecología. Ciudad de México, México, 144 pp.

Yáñez L., García-Nieto E., Rojas E., Carrizales L., Mejía J., Calderón J., Razo I. y Díaz-Barriga F. (2003). DNA damage in blood cells from children exposed to arsenic and lead in a mining area. Environ. Research. 93, 231240. DOI: 10.1016/j.envres.2003.07.005

Zetina-Rodríguez M.C. (2012). La historia de un tesoro que se convirtió en un desastre ambiental, la Zacatecana, ejido de Guadalupe. RCSL 4, 160-194 